\title{
Plasmonic Sensors in the Petro-chemistry World
}

\author{
Francisco Pérez-Ocón* \\ Optics department, University of Granada, Spain \\ *Corresponding author: Francisco Pérez-Ocón, Faculty of Science, Optics department, Granada, Spain.
}

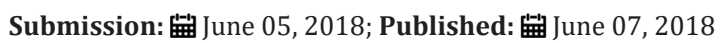

\begin{abstract}
Plasmonic sensors permit us to concentrate light (electromagnetic radiation) in nano-structures. By using these structures, which present superficial plasmonic resonance in the interface of different media, we can manipulate light-material interaction at a nano-technological scale. Their usefulness in the world of petro-chemistry is enormous, we can use them to find out the composition of the chemical compounds in petroleum (without extracting samples), the level of fuel in tanks, etc. in real time by just inserting the sensor into the tanks without any danger because the components of these sensors are made of glass, dielectrics and metal. They work with electromagnetic radiation (usually light) and it is impossible for any explosion to be produced.
\end{abstract}

\section{What is a Plasmonic Sensor?}

In physics, a Plasmon is a quantum of plasma oscillation, it is obtained when the oscillation of the plasma is quantized (such as photons, which are the quantization's of the electromagnetic waves). Another way to define them is to say that plasmon's are oscillations of the density of Fermi's gas (a gas of free electrons) usually with optic frequencies. When a Plasmon interacts with a photon, another cuasi-particular named the polariton of the plasma is created. As plasmon's are the classic quantization of the plasma oscillations, the majority of the properties can be obtained from Maxwell's equations without resorting to quantum mechanics.

Plasmons have an infinite number of applications in industry, especially in the petrochemical industry, particularly for sensors based on surface plasmon resonance (SPR) and these sensors are mostly used in fuel. The structure is based on the configurations of Otto and Kretschmann. Based on the way they work, there are two kinds of sensors, which work either for angle interrogation or for intensity interrogation. We are going to focus on intensity interrogation and the Kretschmann configuration because they are more autonomous, rapid and versatile (Figure 1). Electromagnetic radiation is injected into the optical fiber in the glass hemispherical prism. If a plasmon is generated in the interface dielectric-metal or metal medium, a reduction of the radiation reflection is produced in the interface prism-dielectric and a reduction of the radiation is injected into the exit of the optical fiber, so we are able to detect the substance and the quantity in the medium.

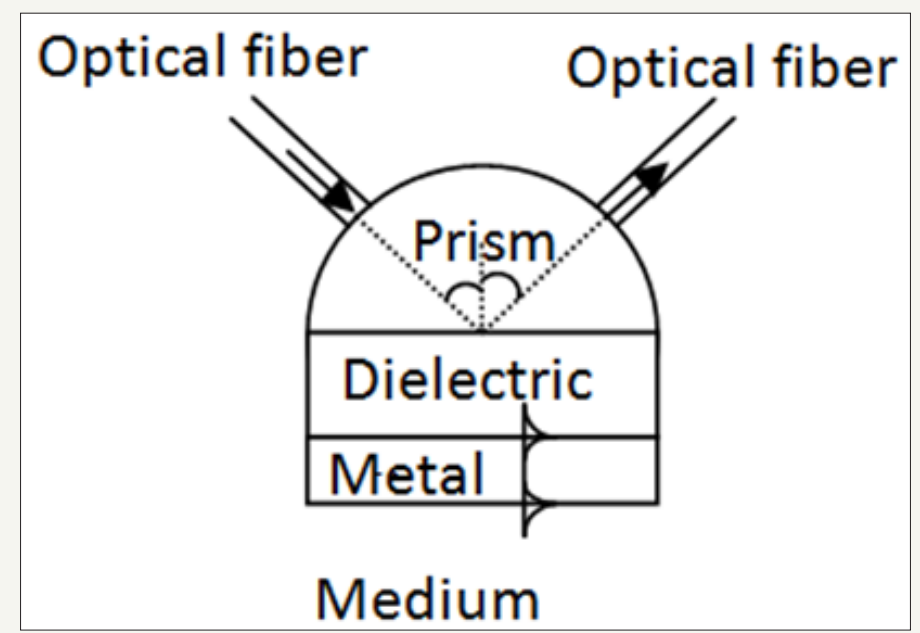

Figure 1: Structure of a plasmonic sensor of intensity interrogation and the Kretschmann configuration. 


\section{What are they for?}

With these sensors we can measure the level of liquids (gasoline, diesel, kerosene, oil, etc.) in any tank. Moreover, we can know whether a substance is mixed in the fuel container (water, sulphur, etc.) to know the quality of the petroleum or whether there are mixtures of different fuels (for instance, petroleum of different qualities). With these sensors, it is no longer necessary to take a sample and analyze it in the laboratory. It is almost impossible (sometimes impossible) to take a sample of some parts of a container, for instance, just from the bottom of the tank without extracting liquids from other parts. With the intensity interrogation plasmonic sensor we can know these results in real time, we can set them in a specific place in any petrol tank or derivate and it is not necessary extract the sample to obtain the information about the composition or, simply, to obtain information about the level of the liquid.

We can install these sensors in a pipe where the fuel is flowing and check if the composition of the fuel is changing (in real time) as the pipe is supplying different tanks, for instance. Currently interrogation plasmonic sensors have a resolution up to 10-7 RIU, i.e., the seventh decimal digit, for this reason, we can detect extremely minute quantities of substances mixed in petroleum, a few parts per million (ppm) for instance. As these liquids are highly flammable, any electrical current (although weak) must be introduced into the flammable or simply conducting liquid. These sensors have a great advantage over other methods as they are non-electrical (without electrical sparks that could cause a fire or explosion in tank) and are also immune to electromagnetic interference. The method is safe and corrosion free, the measurement is highly sensitive, accurate and repeatable. There are no moveable parts (no wear and tear, no problem with replacement pieces), there are no problems of hysteresis (repeatability), and the light is always guided in the same way within the fiber (repeatable and accurate). Plasmonic sensors are the present and the future for the petrochemical industry.
Creative Commons Attribution 4.0

International License

For possible submissions Click Here
Submit Article

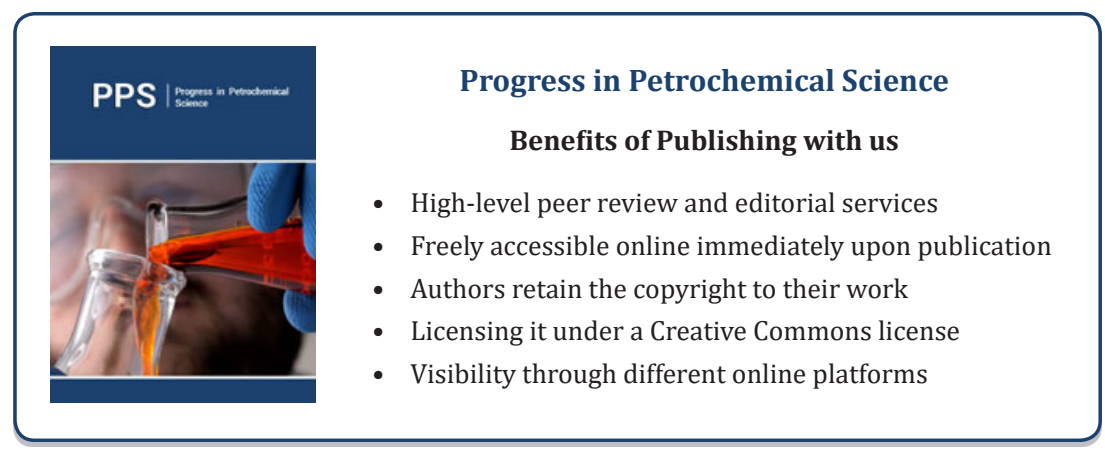

